\title{
Reversible Change of the Spin State in a Manganese Phthalocyanine by Coordination of $\mathrm{CO}$ Molecule
}

\author{
A. Stróżecka, ${ }^{1}$ M. Soriano, ${ }^{2,3}$ J. I. Pascual, ${ }^{1}$ and J. J. Palacios ${ }^{2}$ \\ ${ }^{1}$ Institut für Experimentalphysik, Freie Universität Berlin, 14195 Berlin, Germany \\ ${ }^{2}$ Departamento de Física de la Materia Condensada, Universidad Autónoma de Madrid, \\ Campus de Cantoblanco, 28049 Madrid, Spain \\ ${ }^{3}$ Departamento de Física Aplicada, Universidad de Alicante, 03690 San Vicente del Raspeig, Alicante, Spain
}

(Received 3 May 2012; published 2 October 2012)

\begin{abstract}
We show that the magnetic state of individual manganese phthalocyanine (MnPc) molecules on a $\mathrm{Bi}(110)$ surface is modified when the $\mathrm{Mn} 2+$ center coordinates to $\mathrm{CO}$ molecules adsorbed on top. Using scanning tunneling spectroscopy we identified this change in magnetic properties from the broadening of a Kondo-related zero-bias anomaly when the CO-MnPc complex is formed. The original magnetic state can be recovered by selective desorption of individual $\mathrm{CO}$ molecules. First principles calculations show that the $\mathrm{CO}$ molecule reduces the spin of the adsorbed $\mathrm{MnPc}$ from $S=1$ to $S=1 / 2$ and strongly modifies the respective screening channels, driving a transition from an underscreened Kondo state to a state of mixed valence.
\end{abstract}

Control over the magnetic moment of a molecule and its interaction with a substrate is a key issue in the emerging field of molecular spintronics [1]. In metal-phthalocyanines and metal-porphyrins the metal center is usually coordinatively unsaturated and presents a local reactive site, which opens a unique possibility of controlling the magnetic moment in situ by external chemical stimuli [2-8]. The axial coordination of small molecules like $\mathrm{CO}, \mathrm{NO}$ or $\mathrm{O}_{2}$ to those complexes substantially alters their electronic properties, which has led to a successful implementation of phthalocyanines and porphyrins in gas sensors $[9,10]$. Studies specifically addressing the magnetic state are, however, scarce. Only recently it has been shown that the attachment of a NO molecule to a cobalt-tetraphenylporphyrin (CoTPP) quenches its spin due to the oxidation process [6]. The general picture is however more complicated, as the chemical bond to the reactant molecule causes the redistribution of charge in the $d$ orbitals of the metal center and modifies the ligand field of the metal ion. This has critical consequences for the magnetic ground state of the complex [7,8]. On metal surfaces, the formation of a new ligand bond may additionally alter the hybridization of molecular and substrate states, thus affecting the electronic and magnetic coupling of the metal ion to the substrate [3-5]. Understanding the response of these effects to the change in chemical coordination is crucial to gain full control over the functionality of the magnetic system.

Here, we show that the magnetic moment of a manganese phthalocyanine (MnPc) molecule [see Fig. 1(a)] on a $\mathrm{Bi}(110)$ surface is reduced when coordinated to a $\mathrm{CO}$ molecule. Using a combination of a low temperature scanning tunneling microscopy (STM) and density functional theory (DFT) we find, first, that the spin of the MnPc molecule is reduced from $S=3 / 2$ to $S=1$ upon adsorption and, second, that $\mathrm{CO}$ further reduces the spin of the $\mathrm{MnPc}$ from $S=1$ to $S=1 / 2$. The change in the magnetic ground state upon $\mathrm{CO}$ attachment is detected by the broadening of a characteristic zero-bias anomaly (ZBA). We interpret this broadening as a transition from a Kondo regime, for the bare $\mathrm{MnPc}$ on $\mathrm{Bi}(110)$, to a regime where charge fluctuations drive the $\mathrm{CO}-\mathrm{MnPc}$ complex into a mixed valence state. We further show that the original magnetic state of $\mathrm{MnPc}$ is recovered after tip-induced desorption of the $\mathrm{CO}$ molecule.

Our experiments have been performed in a custom-made STM working in ultrahigh vacuum and at low temperature (5 K). As a semimetal, Bismuth has a low density of states

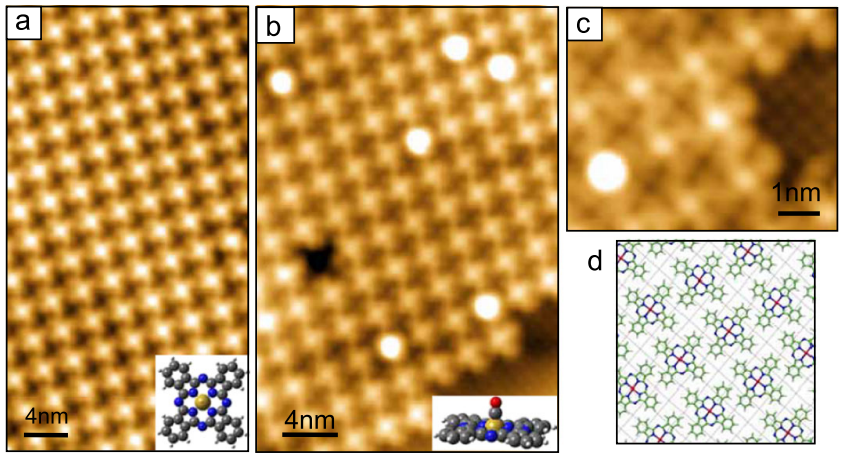

FIG. 1 (color online). Adsorption of MnPc and CO-MnPc on $\mathrm{Bi}(110)$. (a) STM image of a highly ordered MnPc island $(I=0.2 \mathrm{nA}, V=250 \mathrm{mV}$, [44]). (b) MnPc island after exposure to $\mathrm{CO}(I=0.1 \mathrm{nA}, V=180 \mathrm{mV})$. CO-coordinated molecules can be distinguished by different apparent heights. Inset images in (a) and (b) show schematic pictures of the chemical structure of $\mathrm{MnPc}$ and $\mathrm{CO}-\mathrm{MnPc}$. (c) High resolution STM image of bare and $\mathrm{CO}$-ligated $\mathrm{MnPc}(I=0.1 \mathrm{nA}, \quad V=$ $-250 \mathrm{mV}$ ). (d) Adsorption model of MnPc on $\mathrm{Bi}(110)$. 
close to the Fermi level [11], favoring that molecular adsorbates lie weakly affected by the surface [12]. We chose the (110) surface because it additionally presents dangling bonds that can eventually anchor the molecular species to a fixed site, creating a full commensurate layer. We exposed an atomically clean $\mathrm{Bi}(110)$ single crystal surface at room temperature to a flux of $\mathrm{MnPc}$ molecules (Sigma-Aldrich) thermally sublimed in vacuum from a crucible, and posteriorly cool it down for STM inspection.

On this surface $\mathrm{MnPc}$ molecules self-assemble in densely packed islands [Fig. 1(a)]. MnPc molecules appear as a cloverlike protrusion with a bright center corresponding to the metal ion. The adsorption configuration could be established with precision by resolving simultaneously the intramolecular structure of $\mathrm{MnPc}$ and atomic structure of the underlying substrate [see e.g., Fig. 1(c)]. As shown schematically in Fig. 1(d), MnPc adsorbs on Bi(110) with its Mn ion located directly on top of a dangling bond of the surface. This configuration suggests the formation of a local bond between MnPc and the dangling bond of the bismuth atom.

The MnPc covered sample was subsequently annealed up to $130 \mathrm{~K}$ and exposed to $\mathrm{CO}$ gas at partial pressure of $10^{-7}$ mbar for $90 \mathrm{~s}$. After $\mathrm{CO}$ exposure the structure of the molecular islands remains unchanged, but several molecules $(\sim 10 \%)$ now exhibit larger apparent height [Fig. 1(b)]. We identify these new molecules as CO-ligated MnPc. Figure 1(c) shows a high resolution STM image of the new species embedded in the MnPc island. Similarly to $\mathrm{MnPc}$, the CO-coordinated molecules exhibit fourfold symmetry, with a central protrusion appearing $0.8 \AA$ higher than in the case of $\mathrm{MnPc}$, indicating that a single $\mathrm{CO}$ molecule bonds directly to the transition metal ion. We find only singly coordinated $\mathrm{CO}-\mathrm{MnPc}$, contrary to previous studies reporting doubly coordinated porphyrin molecules $[13,14]$.

The differential conductance $(d I / d V)$ spectra measured close to $E_{F}$ reveals a pronounced anomaly at zero bias with a diplike line shape on MnPc [Fig. 2(a)]. We interpret it as a fingerprint of the Kondo effect, as it has been observed before for MnPc and other metal-phthalocyanines on surfaces [15-18]. The zero-bias anomaly exhibits a clear Fano line shape [Figs. 2(a) and 2(b)], commonly observed for Kondo ground states of magnetic adsorbates [19]. Other possible effect which could also cause a zero-bias anomaly, as e.g., inelastic spin flip excitation, have been carefully excluded by studying the temperature dependence of the line shape. A Kondo temperature of $T_{K}^{\mathrm{MnPc}}=22( \pm 6) \mathrm{K}$ can be approximated from a fit to the anomaly's line shape using the Fano equation [20,21].

When similar spectra are measured on $\mathrm{CO}-\mathrm{MnPc}$ molecules, the ZBA appears much broader, with a slightly different line shape [Fig. 2(a)]. The broadening of the zero-bias resonance indicates that the coordination to $\mathrm{CO}$ modifies the magnetic state of the molecule. This modification is
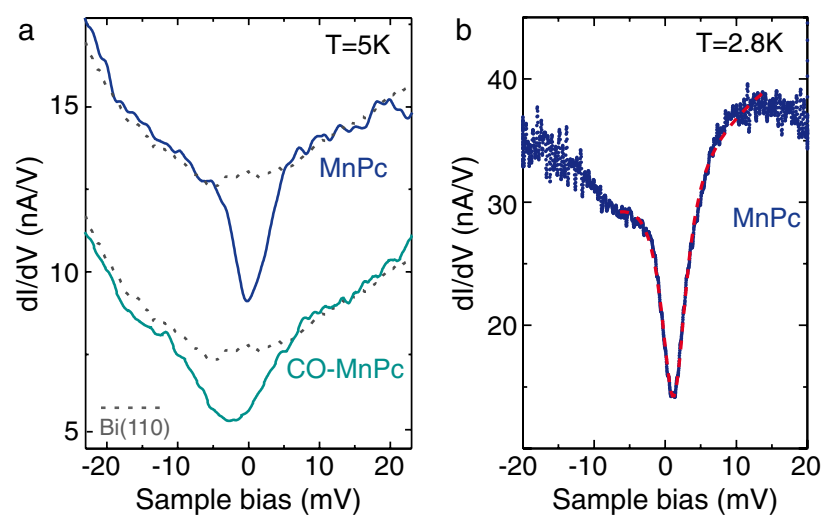

FIG. 2 (color online). (a) $d I / d V$ spectra of MnPc and COcoordinated $\mathrm{MnPc}$ in the bias range close to $E_{F}$, showing the zero bias anomaly. The dotted plots are the spectra measured on a bare $\mathrm{Bi}$ surface as a reference. (b) Zero-bias feature of MnPc measured at reduced temperature $T=2.8 \mathrm{~K}$. The feature exhibits a clear Fano line shape. The dashed line (red) corresponds to the fit by Fano equation.

reversible and can be controlled by selective removal of CO molecules using a STM tip. In order to detach an individual CO from its site, the STM tip was placed over $\mathrm{CO}-\mathrm{MnPc}$ molecule, the feedback loop was opened and the sample bias was ramped. The resulting current-voltage characteristics are shown in Fig. 3(a). A sudden drop of the tunneling current at a certain threshold voltage indicates detachment of the $\mathrm{CO}$ molecule from its coordination
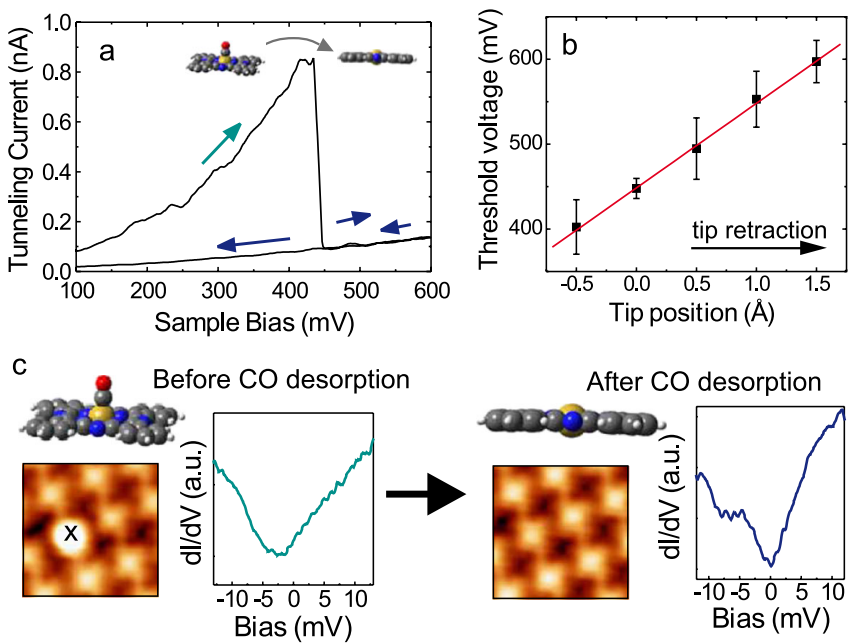

FIG. 3 (color online). Desorption of CO molecules from MnPc islands. (a) $I-V$ characteristics recorded during desorption of $\mathrm{CO}$. A sudden drop in current marks the threshold voltage for $\mathrm{CO}$ detachment. (b) Threshold voltage plot versus tip-sample distance. Zero position of the tip corresponds to the feedback parameters $V_{f}=100 \mathrm{mV}$ and $I_{f}=100 \mathrm{pA}$. Each point is an average of over 30 measurements. Linear fit of the data indicates that the process is driven by electric field of $1 \mathrm{~V} / \mathrm{nm}$. (c) STM image and $d I / d V$ spectrum of the molecule before and after controlled CO desorption. 
site (in most cases to a new site in a neighbor $\mathrm{MnPc}$ molecule). The resulting species recovers the usual clovelike shape and a narrower zero-bias anomaly characteristic of a bare MnPc molecule [Fig. 3(c)]. To establish the underlying mechanism of $\mathrm{CO}$ detachment we note that the threshold voltage increases linearly with the distance between the tip and the molecule, as shown in Fig. 3(b). This behavior indicates that the desorption of $\mathrm{CO}$ is induced by the electric field at the tunnel junction. A critical value of electric field $\varepsilon \cong 1 \mathrm{~V} / \mathrm{nm}$ can be extracted from the slope of the line.

The origin of the zero-bias anomaly on the CO-MnPc molecules cannot be deduced directly from our experiments. One could associate it to a fingerprint of a Kondo ground state with a larger Kondo temperature $\left[T_{K}^{\mathrm{CO}-\mathrm{MnPc}}\right.$ about $50( \pm 10) \mathrm{K}$ can be fitted]. This is, however, unprovable considering the $\pi$-acceptor character of the $\mathrm{CO}$ ligand. As a strong field ligand, $\mathrm{CO}$ affects the magnetic state of MnPc in two ways: (i) it increases the splitting of the $d$ orbitals, and thus favors the low spin configuration in the complex [8]; (ii) it withdraws the electron density from the bond to the ligand in the opposite coordination site (here the $\mathrm{Bi}$ dangling bond) and hence reduces the coupling of the $d$ orbitals to the substrate [2-4]. The latter effect, known in coordination chemistry as trans effect, should reduce the Kondo temperature, contrary to our experimental observation.

To rationalize the experimental results we performed DFT calculations of free and adsorbed $\mathrm{MnPc}$ and $\mathrm{CO}-$ MnPc molecules. All calculations were performed using the Green's function formalism implemented in our code ANT.G, which interfaces with GAUSSIAN03/09 [22,23]. We used a general gradient approximation (GGA) with the Becke and Perdew-Becke-Ernzerhof exchange-correlation functional [24] in combination with a common double local basis set LANL2DZ [25,26]. Different views of the computed system are shown in Fig. 4(a). The Bi(110) surface was described using two bilayers embedded on a tight-binding Bethe lattice. Following the experimental results (Fig. 1), $\mathrm{MnPc}$ and $\mathrm{CO}-\mathrm{MnPc}$ molecules were placed with the manganese atom directly on top of a $\mathrm{Bi}$ atom with an optimized distance to the surface and rotated seven degrees with respect to the high symmetry axis of the $\mathrm{Bi}(110)$ surface. In this configuration, four carbon atoms of the phthalocyanine cycle and the central $\mathrm{Mn}$ ion are in direct contact with $\mathrm{Bi}(110)$ dangling bonds.

We simulate first the electronic and spin configuration of the free $\mathrm{MnPc}$ molecule, obtaining results in close agreement with previous studies [27-29]. The molecule has a spin state $S=3 / 2$ due to three unpaired electrons localized on the manganese orbitals $d_{x z} / d_{y z}, d_{z^{2}}$, and $d_{x y}$. The $d_{x z} / d_{y z}$ orbitals, hosting three electrons, are strongly hybridized with the phthalocyanine ring of the molecule, masking the atomic character of its localized spin. The $d_{x y}$ orbital hosts the most localized unpaired electron.
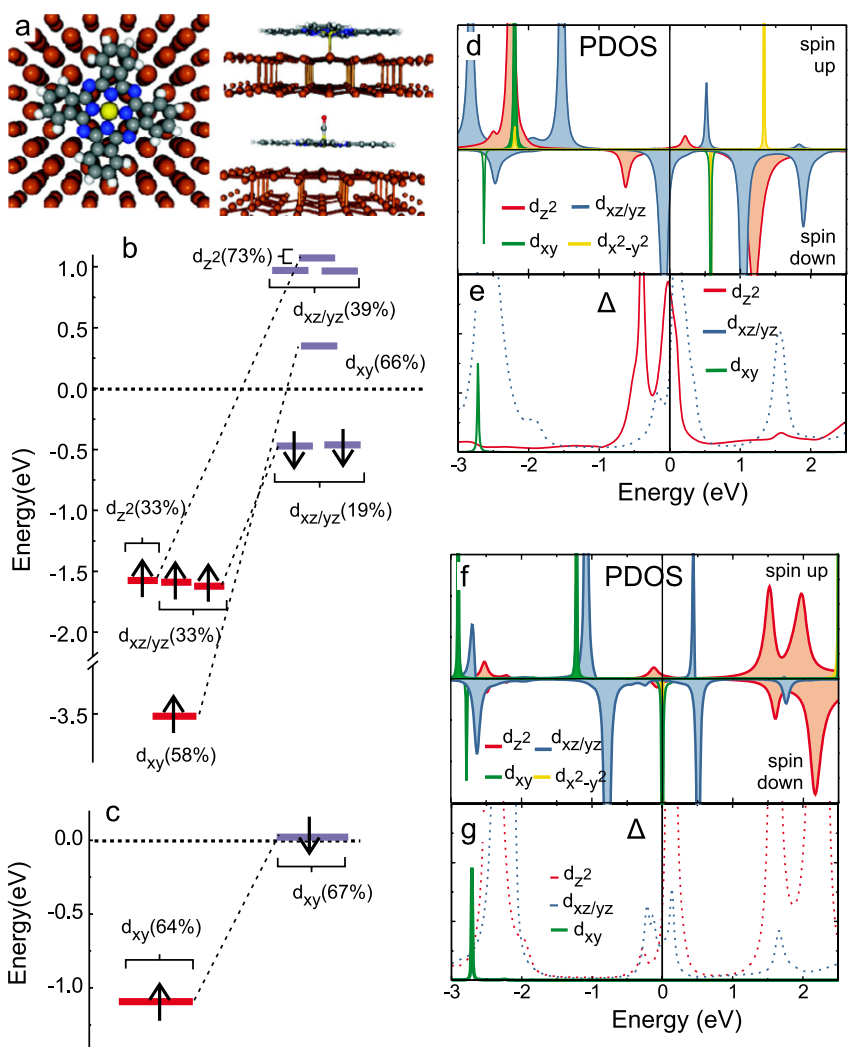

FIG. 4 (color online). (a) Model of computed system: top view (left) and side view (right) of $\mathrm{CO}-\mathrm{MnPc}$ (top) and $\mathrm{MnPc}$ (bottom). (b),(c) Energy spectrum of adsorbed MnPc (b) and $\mathrm{CO}-\mathrm{MnPc}$ (c), showing the relevant spin states. Bars in left (right) show the up (down) eigenvalues. The rate in parentheses gives the atomic character of the molecular orbital. (d),(f) Local density of states (PDOS) and (e), (g) hybridization function $(\Delta)$ projected on $d$ orbitals of adsorbed $\mathrm{MnPc}(\mathrm{d}),(\mathrm{e})$ and $\mathrm{CO}-\mathrm{MnPc}$ (f),(g). The dotted lines in (e) and (g) refer to the orbitals which are not relevant for the magnetism of the molecule.

On the $\mathrm{Bi}(110)$ surface, $\mathrm{MnPc}$ keeps two unpaired electrons in the $d$ orbitals. The net spin density in the $d_{x z}$ and $d_{y z}$ orbitals is reduced and now results from four occupied molecular orbitals with different atomic contribution depending on the spin orientation [Fig. 4(b)]. In addition, a spin polarization arises in the ligands, opposite to that in the Mn core. Thus, the total spin is reduced to $S=1$, with two identifiable and localized unpaired electrons in the the $d_{z^{2}}$ and $d_{x y}$ orbitals [Fig. 4(b)]. We also find that a spin polarized density arises on $\mathrm{Bi}$ atoms directly underneath the adsorbed molecule, antiferromagnetically coupled to the spin on the Mn core and thus supporting the emergence of Kondo screening.

The strength of a Kondo screening channel depends, to a first approximation, on the hybridization with the substrate. For $\mathrm{MnPc}$ on $\mathrm{Bi}(110)$, the spin-polarized local density of states (LDOS) projected on the Mn- $d$ orbitals shows that each orbital hosting an unpaired electron interacts differently with the surface [Fig. 4(d)]. The Mn- $d_{z^{2}}$ orbital 
hybridizes with a dangling bond of $\mathrm{Bi}$ and broadens substantially. The $d_{x y}$ orbital appears as narrow peaks in the PDOS spectrum. This indicates that this state remains highly decoupled from the surface, probably to the absence of the $z$ component. The result is that the two unpaired spins are screened via Kondo screening channels with a very different energy scale and, thus, Kondo temperature [17]. Such a scheme suggests an underscreened spin if the experimental temperature lies between the Kondo temperatures of each screening channels [30,31].

In the DFT framework, the orbital-substrate coupling is represented by a hybridization function which allows for a qualitative analysis of the feasible Kondo channels [32,33]. The hybridization function is given by $\boldsymbol{\Sigma}_{\mathbf{d}}(\omega)=$ $(\omega+\mu) \mathbf{I}-\mathbf{H}_{\mathbf{d}}^{\mathbf{K S}}-\left(\mathbf{G}_{\mathbf{d}}(\omega)\right)^{-1}$, where $\omega$ and $\mu$ are the energy and the chemical potential, respectively, $\mathbf{I}$ is the identity matrix, $\mathbf{H}_{\mathbf{d}}^{\mathbf{K S}}$ is the Kohn-Sham Hamiltonian and $\mathbf{G}_{\mathbf{d}}$ is the one electron Green's function projected onto the $d$-subshell. Only the imaginary part of the hybridization function is relevant in this work $\left(\Delta=-\operatorname{Im} \Sigma_{\mathbf{d}}(\omega) / \pi\right)$. Specifically, the prerequisite for the Kondo effect of the host orbital having a finite coupling to the electron bath is equivalent to having nonzero value of the hybridization function $\Delta$ at the Fermi level [34]. The value of $\Delta$ calculated for different $\mathrm{Mn} d$ orbitals of adsorbed MnPc is shown in Fig. 4(g). The $d_{z^{2}}$ orbital shows a peak in the hybridization function at the Fermi level indicating that this orbital is strongly screened by the substrate. On the contrary, the hybridization function of the $d_{x y}$ orbital has a zero value in a broad energy window in the vicinity of the Fermi level. These results suggest that the Fano resonance in the spectra of the MnPc molecule is a fingerprint of a Kondo channel opened to screen the $d_{z^{2}}$ orbital. The unpaired spin of the $d_{x y}$ orbital remains decoupled from the electron bath and this Kondo channel is closed. On the basis of these simulations, we conclude that the MnPc molecule retains part of its magnetic moment on the surface, lying in an underscreened many-body ground state. This is a consequence of the huge disparity in hybridization functions between spin channels, making this system unique in this regard.

Next, we perform a similar analysis for the CO-MnPc molecular complex. The $\mathrm{CO}$ molecule stays perpendicular to the MnPc molecular plane [Fig. 4(a)]. It is anchored to the manganese ion via its carbon atom through a synergic $\pi^{*}$ backbonding, which strongly modifies the electron population and the spin of the Mn $d$ orbitals in the following manner. The $d_{z^{2}}$ and the $d_{x z} / d_{y z}$ orbitals lose their magnetic moment: the former is emptied due to the overlap with a nonbonding orbital of $\mathrm{CO}$ and the latter is fully occupied due to the bond with the $p_{x}$ and the $p_{y}$ orbitals of CO. The spin-resolved LDOS of the adsorbed CO-MnPc projected on the Mn $d$ orbitals [Fig. 4(f)] confirms that the spin-polarization of the $d_{z^{2}}$ and $d_{x z} / d_{y z}$ orbitals is reduced. Only the $d_{x y}$ orbital appears to be relevant for the magnetism of the complex. The total spin of the MnPcCO complex is reduced to $S=1 / 2$, stemming from an unpaired electron localized in a molecular orbital with strong atomic $d_{x y}$-orbital character but a finite contribution from the phthalocyanine cycle [see Fig. 4(c)].

It thus appears clear that the modification of the experimentally observed Kondo resonance upon CO coordination is a direct consequence of the redistribution of the $d$ electrons of the Mn atom. However, the ZBA broadening cannot be understood as an increase in the Kondo screening because the responsible level is very localized. As seen in Fig. $4(\mathrm{~g})$, the $d_{x y}$ hybridization function is zero in the vicinity of the Fermi level, which confirms that also now no Kondo effect can be mediated by this orbital.

This apparent inconsistency with the experimental observations can be bypassed by noting that the PDOS on the $d_{x y}$ orbital shows a highly localized branch pinned at the Fermi level. This state thus belongs to the lowest unoccupied orbital of the molecule (LUMO), and is the only one responsible for the chemical potential lineup between the molecule and the substrate [35]. In these circumstances, charge fluctuations are expected to occur at the $d_{x y}$ orbital. When electronic correlations are considered, a mixedvalence regime is likely to emerge, as it is known for the $f$-electron compounds [34]. In this situation, one expects a resonance to appear in the differential conductance spectra at zero bias, similar to that in the Kondo regime but characterized by a larger width [36,37]. It is interesting to note that the small hybridization with the surface prevents the finite spin in the $d_{x y}$ orbital from quenching, in contrast with the scenario reported in related systems $[38,39]$. This allows for a many-body correlated state to emerge.

Finally, we comment on the possible role of the surface states in the screening process on $\mathrm{Bi}(110)$. As a semimetal, bismuth is characterized by a low density of bulk states at the Fermi level; its surfaces are, however, strongly metallic due to the presence of the surface states. Therefore, the Kondo effect on bismuth can be expected to involve surface states rather than bulk states. However, strong spinorbit coupling induces the spin polarization of the surface states through the Rahsba effect [11]; the concept of screening in such system is not straightforward [40]. The Kondo effect involving the chiral spin states has been recently intensively discussed in the context of topological insulators [41-43] and can be understood assuming a complex structure of the Kondo cloud with a nontrivial spatial and spin dependence [43].

In summary, we have demonstrated that the magnetic ground state of individual metal-organic molecules can be reversibly modified by chemical coordination to external molecular species acting as ligands. Using a combination of low-energy scanning tunneling spectra and $a b$ initio calculations, we find that the bonding of a $\mathrm{CO}$ molecule to the $\mathrm{Mn}^{2+}$ center of MnPc molecules on $\mathrm{Bi}(110)$ leads to 
a substantial modification of its spin state. Our results indicate that the chemically induced magnetic transition corresponds to a change from a high-spin underscreened Kondo state to a mixed-valence state, where charge fluctuations destroy the spin $1 / 2$ state of the CO-MnPc complex. Our results thus emphasize the ultimate limits of manipulating magnetic states of individual molecules by using a combination of tunneling electrons and chemical control over the molecular spin, which could facilitate molecular scale patterning of magnetic motifs in molecular thin films.

We thank K. J. Franke, B. W. Heinrich, and D. Jacob for fruitful discussions. This research was supported by Deutsche Forschungsgemeinschaft (DFG-STR 1151/1-1 and SFB 658) and by MICINN under Grants No. FIS2010-21883 and No. CONSOLIDER CSD20070010. M. S. acknowledges computational support from the CCC of the Universidad Autónoma de Madrid.

[1] L. Bogani and W. Wernsdorfer, Nature Mater. 7, 179 (2008).

[2] J. M. Gottfried and H. Marbach, Z. Phys. Chem. 223, 53 (2009).

[3] K. Flechtner, A. Kretschmann, H. P. Steinrück, and J. M. Gottfried, J. Am. Chem. Soc. 129, 12110 (2007).

[4] W. Hieringer, K. Flechtner, A. Kretschmann, K. Seufert, W. Auwaerter, J. V. Barth, A. Goerling, H.-P. Steinrück, and J. M. Gottfried, J. Am. Chem. Soc. 133, 6206 (2011).

[5] J. Miguel, C.F. Hermanns, M. Bernien, A. Krueger, and W. Kuch, J. Phys. Chem. Lett. 2, 1455 (2011).

[6] C. Wäckerlin, D. Chylarecka, A. Kleibert, K. Muller, C. Iacovita, F. Nolting, T. A. Jung, and N. Ballav, Nature Commun. 1, 1 (2010).

[7] C. Isvoranu, B. Wang, E. Ataman, K. Schulte, J. Knudsen, J. N. Andersen, M.-L. Bocquet, and J. Schnadt, J. Chem. Phys. 134, 114710 (2011).

[8] C. Isvoranu, B. Wang, E. Ataman, J. Knudsen, K. Schulte, J. N. Andersen, M.-L. Bocquet, and J. Schnadt, J. Phys. Chem. C 115, 24718 (2011).

[9] G. Guillaud, J. Simon, and J. P. Germain, Coord. Chem. Rev. 178, 1433 (1998).

[10] F. I. Bohrer, A. Sharoni, C. Colesniuc, J. Park, I. K. Schuller, A.C. Kummel, and W.C. Trogler, J. Am. Chem. Soc. 129, 5640 (2007).

[11] Ph. Hofmann, Prog. Surf. Sci. 81, 191 (2006).

[12] G. Schulze, K. J. Franke, and J. I. Pascual, Phys. Rev. Lett. 109, 026102 (2012).

[13] K. Seufert, M.-L. Bocquet, W. Auwaerter, A. WeberBargioni, J. Reichert, N. Lorente, and J. V. Barth, Nat. Chem. 3, 114 (2011).

[14] K. Seufert, W. Auwaerter, and J. V. Barth, J. Am. Chem. Soc. 132, 18141 (2010).

[15] Y. S. Fu et al., Phys. Rev. Lett. 99, 256601 (2007).

[16] L. Gao et al., Phys. Rev. Lett. 99, 106402 (2007).

[17] K. J. Franke, G. Schulze, and J. I. Pascual, Science 332, 940 (2011).
[18] A. Mugarza, C. Krull, R. Robles, S. Stepanow, G. Ceballos, and P. Gambardella, Nature Commun. 2, 490 (2011).

[19] O. Ujsaghy, J. Kroha, L. Szunyogh, and A. Zawadowski, Phys. Rev. Lett. 85, 2557 (2000).

[20] K. Nagaoka, T. Jamneala, M. Grobis, and M. F. Crommie, Phys. Rev. Lett. 88, 077205 (2002).

[21] Thermal and instrumental broadening has been removed.

[22] M. Frisch, G. W. Trucks, H. B. Schlegel, G. E. Scuseria, M. A. Robb, J.R. Cheeseman, J. A. Montgomery, J. T. Vreven, and K. N. Kudin, GAUSSIAN03. Revision B.01. (2003).

[23] J. J. Palacios, D. Jacob, A. J. Perez-Jimenez, E. S. Fabian, E. Louis, and J. A. Vergs, ALACANT. Ab-Initio Quantum Transport Package, http://alacant.dfa.ua.es.

[24] J. P. Perdew, K. Burke, and M. Ernzerhof, Phys. Rev. Lett. 77, 3865 (1996).

[25] T.H. Dunning and P. J. Hay, in Methods of Electronic Structure Theory, edited by H. F. Schaefer (Plenum, New York, 1977), p. 1.

[26] W. R. Wadt and P. J. Hay, J. Chem. Phys. 82, 284 (1985).

[27] J. Wang, Y. Shi, J. Cao, and R. Wu, Appl. Phys. Lett. 94, 122502 (2009).

[28] X. Shen, L. Sun, E. Benassi, Z. Shen, X. Zhao, S. Sanvito, and S. Hou, J. Chem. Phys. 132, 054703 (2010).

[29] M. Grobosch, B. Mahns, C. Loose, R. Friedrich, C. Schmidt, J. Kortus, and M. Knupfer, Chem. Phys. Lett. 505, 122 (2011).

[30] A. Posazhennikova, B. Bayani, and P. Coleman, Phys. Rev. B 75, 245329 (2007).

[31] R. Korytar and N. Lorente, J. Phys. Condens. Matter 23, 355009 (2011).

[32] D. Jacob, K. Haule, and G. Kotliar, Phys. Rev. B 82, 195115 (2010).

[33] M. Karolak, D. Jacob, and A. I. Lichtenstein, Phys. Rev. Lett. 107, 146604 (2011).

[34] A. C. Hewson, in The Kondo Problem to Heavy Fermions (Cambridge University Press, Cambridge, England, 1993).

[35] H. Ishii, K. Sugiyama, E. Ito, and K. Seki, Adv. Mater. 11, 605 (1999).

[36] T. Costi, A. Hewson, and V. Zlatič, J. Phys. Condens. Matter 6, 2519 (1994).

[37] D. Goldhaber-Gordon, J. Göres, M. A. Kastner, H. Shtrikman, D. Mahalu, and U. Meirav, Phys. Rev. Lett. 81, 5225 (1998).

[38] S. Stepanow, P. S. Miedema, A. Mugarza, G. Ceballos, P. Moras, J. C. Cezar, C. Carbone, F. M. F. de Groot, and P. Gambardella, Phys. Rev. B 83, 220401 (2011).

[39] A. Mugarza, R. Robles, C. Krull, R. Korytar, N. Lorente, and P. Gambardella, Phys. Rev. B 85, 155437 (2012).

[40] M. Zarea, S. E. Ulloa, and N. Sandler, Phys. Rev. Lett. 108, 046601 (2012).

[41] J. J. Cha, J. R. Williams, D. S. Kong, S. Meister, H. L. Peng, A. J. Bestwick, P. Gallagher, D. GoldhaberGordon, and Y. Cui, Nano Lett. 10, 1076 (2010).

[42] X.-Y. Feng, W. Q. Chen, J.H. Gao, Q. H. Wang, and F.-C. Zhang, Phys. Rev. B 81, 235411 (2010).

[43] R. Žitko, Phys. Rev. B 81, 241414 (2010).

[44] I. Horcas, R. Fernandez, J. M. Gomez-Rodriguez, J. Colchero, J. Gomez-Herrero and A. M. Baro, Rev. Sci. Instrum. 78, 013705 (2007). 